\title{
Regularization and Renormalization of Quantum Field Theories on Noncommutative Spaces
}

\author{
Harald GROSSE ${ }^{\dagger}$ and Raimar WULKENHAAR $\ddagger$ \\ $\dagger$ Institut für Theoretische Physik der Universität Wien, \\ Boltzmanngasse 5, A-1090 Wien, Austria \\ E-mail: harald.grosse@univie.ac.at \\ $\ddagger$ Max-Planck-Institut für Mathematik in den Naturwissenschaften, \\ Inselstraße 22-26, D-04103 Leipzig, Germany \\ E-mail: raimar.wulkenhaar@mis.mpg.de
}

This article is part of the Proceedings titled "Geometrical Mathods in Physics: Bialowieza XXI and XXII"

\begin{abstract}
We first review regularization methods based on matrix geometry which provide an ultraviolet cut-off for scalar fields respecting the symmetries. Sections of bundles over the sphere can be quantized, too. This procedure even allows to regularize supersymmetry without violating it. Recently, this work was extended to include quantum group covariant regularizations.

In a second part recent attempts to renormalize four-dimensional deformed quantum field theory models are reviewed. For scalar models the well-known UV/IR-mixing does not allow to use standard techniques. The same applies to the Yang-Mills model in four dimensions. Only additional symmetry, as it occurs in the Wess-Zumino model, may allow to avoid this problem.

There was some hope that circumventing UV/IR-mixing by application of the Seiberg-Witten map one can achieve renormalizability of noncommutative field theories. Although the photon self-energy is renormalizable to all orders, $\theta$-expanded noncommutative QED was shown to be not renormalizable, thus ruling out this approach as well.
\end{abstract}

\section{Introduction}

More than ten years ago, John Madore introduced what he called the Fuzzy Sphere. If you work within a geometry which does not have points, singularities of quantum field theory models may be cured. We applied these ideas first to simple two-dimensional models [1].

Later on, together with Klimčik and Presnajder we treated all kind of models from scalar fields to spinor and gauge fields, even supersymmetric models were successfully treated. We also dealt with models defined on $C P(2)$, on a cylinder and on a hyperboloid. All this was within what is now called the Lie algebra deformation. 
Recently, together with John Madore and Harold Steinacker we went to $q$-deformed models. We obtained a cut-off procedure based on a sequence of embedded Podles spheres and were able to formulate a differential calculus which led to gauge models, too. In this way we connected the Yang-Mills models and the Chern-Simons model to those obtained from string theory.

Despite many attempts, four-dimensional quantum field theory on commutative spacetime is still in bad shape. For two-dimensional models constructive methods as well as new algebraic methods led to enormous insights. Even three-dimensional models are quite well understood. In four dimensions we have to rely on renormalized perturbation theory, and attempts to cure the diseases go from unification ideas to adding additional dimensions, strings, etc. It is natural therefore to ask for alternatives.

One idea is to change the geometry. The final goal would be to include full quantized gravity, but on the way we may well study quantum field theoretic models on quantized space-time. This led recently to some surprises and new problems have shown up.

There is an old and simple argument that a smooth space-time manifold contradicts quantum physics. In order to localize an event within a region of extension $l$ one has to transfer an energy of the order $h c / l$. This energy generates a gravitational field. A strong gravity field prevents on the other hand signals to reach an observer. If one inserts the energy density to the rhs of Einstein's equation and puts a length $r$ characterizing the curvature of space-time we get

$$
1 / r^{2}=\left(G / c^{4}\right)(h c / l)\left(1 / l^{3}\right) .
$$

Next we put the two length scales to be equal, because it is certainly operational impossible to localize an event beyond this resulting Planck length. To the best of our knowledge, the first time this argument was put to precise mathematics was in the work by Doplicher, Fredenhagen and Roberts [2]. They obtained what is now called the canonical deformation but averaged over two-spheres. We believe that this averaging will not improve the divergence problems discussed below, although explicit calculations have not been performed so far.

Our work is guided by noncommutative geometry, see $[3,4,5]$ for books giving an overview. We first replace the algebra of functions over a manifold by a deformed noncommutative algebra. Three kinds of deformations are treated. If the commutator of coordinates is put equal to a constant antisymmetric tensor, we call it the canonical deformation. If it is put to be a linear function of coordinates we call it a Lie algebra deformation, and if it is put to be a quadratic expression we call it a quantum group deformation. In the last case the Yang-Baxter equation has to be fulfilled.

Next step concerns the differential calculus. We replace vector fields by derivations on the algebra, we define differential forms by duality. Although it is somewhat tricky to do this for the $q$-deformed sphere, in all three cases one succeeds. Hodge duality, Lie derivatives, the Laplace operator etc, in summary most of the steps of ordinary differential geometry can be simulated.

An essential step concerns fields. Classically they are sections of bundles, but also modules over the algebra. This last notion can be taken over to the noncommutative situation. We study finitely generated projective modules over the deformed algebra and are able to quantize scalar, spinor and gauge fields. 
Finally we write down actions and integrate over the algebra, so we use certain trace functionals. Next steps concern cohomology problems, the formulation of the spectral triples with the help of a Dirac operator and the use of cyclic cocyles. The final version of Alain Connes' program [3] concerns the spectral action which allows to unify all interactions of the standard model and general relativity within one principle [6].

\section{Regularization of Quantum Field Theory}

\subsection{Scalar Fields}

The ideas of deforming or quantizing the commutative algebra of functions over a manifold can best be explained for the simple example of the two-sphere, the deformation of which leads to the Fuzzy Sphere. The Euclidean action of a scalar field is given by

$$
S[\Phi]=\frac{1}{4 \pi} \int_{S^{2}} d \Omega\left[\left(J_{i} \Phi\right)^{2}+\mu^{2} \Phi^{2}+\operatorname{POL}(\Phi)\right]
$$

and is invariant under isometries or rotations of the sphere. The generators $J_{i}$ are angular momentum operators, they close under $s u(2)$. The field $\Phi$ can be expanded according to the infinite set of irreducible representations of $s u(2)$.

Next we truncate this tower: Consider vector spaces transforming according to the first $N$ representations. They can be identified with mappings from the representation space $N / 2$ to itself. These mappings are $(N+1) \times(N+1)$-dimensional matrices, the noncommutative product of these is taken as the product within the algebra. In addition we have to give a precise description of the embedding of these algebras for different $N$, which gives a precise meaning also to the limit. For details see $[7,8,9]$. The Lie algebra of the generators of this algebra is easy to describe. They form the $s u(2)$ Lie algebra with suitable rescaling, such that the Casimir operator still fulfills the defining relation for the two-sphere as an operator:

$$
\begin{aligned}
& {\left[\hat{X}_{i}, \hat{X}_{j}\right]=\mathrm{i} \lambda \varepsilon_{i j k} \hat{X}_{k}, \quad \sum_{i=1}^{3} \hat{X}_{i}^{2}=R^{2},} \\
& R \lambda^{-1}=\sqrt{\frac{N}{2}\left(\frac{N}{2}+1\right)} .
\end{aligned}
$$

Since we work on a matrix algebra it is easy to introduce a differential calculus. All derivations are inner, they are given by the adjoint action with the generators themselves. For the two-sphere we take the generators introduced above and can develop a derivationbased differential calculus. Our aim next is to make sense out of a functional integral of the type

$$
\langle F[\Phi]\rangle=\frac{\int D \Phi \mathrm{e}^{-S[\Phi]} F[\Phi]}{\int D \Phi \mathrm{e}^{-S[\Phi]}} .
$$

The action (2.1) will be replaced by

$$
S[\phi]=(1 / N) \operatorname{Tr}\left(\left[\hat{X}_{i}, \Phi\right]\left[\hat{X}_{i}, \Phi\right]+\operatorname{POL}(\Phi)\right),
$$


where everything depends on $N$. As for the measure we take just the product measure for the finite number of degrees of freedom. This makes the functional integral well-defined.

In the limit where $N$ tends to infinity, the old divergences show up. Feynman rules can be developed, a tadpole graph for the $\Phi^{4}$ model diverges logarithmically in the limit.

In the same spirit it is possible to quantize Kähler manifolds like $C P(n)$. Models on the cylinder and on hyperboloids have been treated, too.

In addition it is possible to quantize sections of line bundles over $S^{2}$, which are characterized by the Chern number. In this way we construct projective modules which lead in a certain limit to the sections of the line bundles over $S^{2}$. We start from the Hopf fibration of $S^{3}$ over $S^{2}$ with fiber $U(1)$. Let $\chi_{1}$ and $\chi_{2}$ be components of a spinor. Restrict the sum of the squares of these two complex numbers to be $R$. This defines the sphere $S^{3}$. Next we study expansions in terms of these two complex coordinates and their complex conjugates. Define $X_{i}=\chi^{\dagger} \sigma_{i} \chi$. The squares of $X_{i}$ sum up to $R^{2}$ and define therefore $S^{2}$. If in an expansion an equal number of $\chi^{\prime}$ 's and $\chi^{\dagger}$ 's occur, it becomes a well-defined function on $S^{2}$. Otherwise it is a section of a bundle.

Next we quantize this scheme: Replace the complex quantities by creation and annihilation operators of two bosons:

$$
\left[A_{i}, A_{j}^{\dagger}\right]=\delta_{i j}, \quad i, j=1,2 .
$$

This means we use the Jordan-Schwinger representation of $s u(2)$. Next we define $N$ dimensional subspaces $F_{N}$ of the Fock space given by a fixed number of $N-1$ creation operators. They form an irreducible representation of $s u(2)$. We study now maps from one such subspace to another one. If they are of equal dimension, square matrices will map one to the other and the sequence of them forms again the Fuzzy Sphere. If they are of different dimensions and their difference equals twice the topological charge, we quantize in a certain sense sections of the bundles. This way we obtain a sequence of embedded modules formed from non-square matrices of special size.

The Dirac operator and spinors were obtained from a supersymmetric treatment. We extended $s u(2)$ to the supergroup $\operatorname{ssp}(2 / 1)$ and obtained a quantization of superfields through an embedded sequence of graded matrix algebras.

\subsection{The Fuzzy $q$-Sphere}

The above described Fuzzy Sphere is invariant under the action of $S O(3)$, or equivalently under the action of $U(s o(3))$. Together with John Madore and Harold Steinacker we defined a sequence of finite algebras, which have analogous properties, but which are covariant under the quantized universal enveloping algebra $U_{q}(s u(2))[10,11]$. This has been done for real $q$ as well as for $q$ being a root of unity. In the latter case certain restrictions have to be obeyed. Covariance of an algebra $A$ under $U_{q}(s u(2))$ means that there exists an action

$$
U_{q}(s u(2)) \times A \rightarrow A, \quad(u, a) \mapsto u \triangleright a,
$$

such that $u \triangleright(a b)=\left(u_{(1)} \triangleright a\right)\left(u_{(2)} \triangleright b\right)$ for $a, b \in A$. Here $\Delta(u)=u_{(1)} \otimes u_{(2)}$ is the Sweedler notation for the coproduct. 
We may follow now the undeformed scheme and define $q$-deformed creation and annihilation operators:

$$
A^{\dagger i} A_{j}=\delta_{j}^{i}+q \hat{R_{j l}^{i k}} A_{k} A^{\dagger l} .
$$

Next one considers again $N$-dimensional subspaces of the Fock space which form irreducible representations of $U_{q}(s u(2))$. It is possible to define the $q$-deformed spheres in terms of $Z_{i}=A^{\dagger \alpha} \epsilon_{\alpha \beta} \sigma_{i}^{\beta \gamma} A_{\gamma}$ by

$$
\epsilon_{k}^{i j} Z_{i} Z_{j}=\text { constant } \cdot Z_{k}
$$

and to prove that the full matrix algebra is generated. After a study of the reality structure we introduced an invariant integral and studied a differential calculus. There exists a unique three-dimensional module of one-forms. As opposed to the classical case, an additional radial one-form shows up. This leads to the addition of a scalar field.

Next it is possible to write down actions for scalar fields as well as for gauge fields. Three possible actions can be formed for the latter:

$$
\begin{aligned}
& S_{1}=\int A * A+2 A \Theta, \\
& S_{2}=2 \int A^{3}+3\left(A d A+A\left(^{*} A\right)\right)+6 A \Theta+2 \Theta^{3}, \\
& S_{3}=\int\left(d A+A^{2}\right)\left(*\left(d A+A^{2}\right)\right),
\end{aligned}
$$

were ${ }^{*}$ denotes the Hodge star operation and $\Theta$ a special one-form which plays the rôle of the Maurer-Cartan form. The commutator of algebra elements with this special form gives one-forms:

$$
d f=[\Theta, f] .
$$

As for the step from one-forms to two-forms one has to take the commutator but subtract the Hodge star of the one form in order to get two-forms,

$$
d \alpha=[\Theta, \alpha]_{+}-{ }^{*} \alpha,
$$

where the anticommutator enters. The step to three-forms is given again by the commutator with this special form.

It is interesting to note that special linear combinations of $S_{i}$ 's correspond to the YangMills action and to the Chern-Simons action. A special combination of both resulted from string theory after taking a particular limit [12].

Very recently we studied second quantization of a field theory on the $q$-deformed fuzzy sphere for $q$ real. For this case it was necessary to perform a path integral over modes, which generate a quasi-associative algebra. This way we kept the symmetry and obtained a smooth limit for $q$ going to 1 . 


\section{Renormalization of deformed quantum field theory}

\section{$3.1 \quad$ UV/IR-mixing}

Inspired to a large extent by the work of Seiberg and Witten [13], numerous investigations of field theories on deformed space(-time) have appeared. The deformed space under consideration - called noncommutative $\mathbb{R}^{4}$ — can be introduced in several ways. First, in analogy to quantum mechanics one assumes that the four-dimensional coordinate operators obey a commutator relation like

$$
\left[x^{\mu}, x^{\nu}\right]=\mathrm{i} \theta^{\mu \nu}=-\mathrm{i} \theta^{\nu \mu} .
$$

Thanks to the Baker-Campbell-Hausdorff formula, plane waves $u_{p}=\mathrm{e}^{\mathrm{i} p_{\mu} x^{\mu}}$ obey the algebra

$$
u_{p} u_{q}=\mathrm{e}^{-\frac{1}{2} \mathrm{i}^{\mu \nu} p_{\mu} q_{\nu}} u_{p+q}
$$

were $p$ and $q$ denote commutative $d$-dimensional momenta. Derivations on this algebra are defined through the multiplication with these momenta, an integral on this algebra is defined by mapping to the $p=0$ part. In the form (3.2) we make contact to the noncommutative torus, where $p$ and $q$ run over an integer lattice. The latter occurred through compactification of M-theory on noncommutative 2-tori [14] and corresponds to turning on a background three-form.

Superpositions of plane waves with smooth functions $f(x)$ gives the Weyl operators $W[f]$. A standard question is which product of functions allows to encode this algebra. The answer is given by the Moyal-Weyl product

$$
W[f] W[g]=W[f \star g], \quad(f \star g)(x)=\left.\mathrm{e}^{\frac{1}{2} \mathrm{i} \theta^{\mu \nu} \partial_{\mu}^{y} \partial_{\nu}^{z}} f(y) g(z)\right|_{x=y=z} .
$$

Unless the functions $f, g$ are analytic, the derivatives are meant in a distributional sense. For Schwartz class functions a useful formula is

$$
(f \star g)(x)=\int \frac{d^{4} k}{(2 \pi)^{4}} \int d^{4} y f\left(x+\frac{1}{2} \theta \cdot k\right) g(x+y) \mathrm{e}^{\mathrm{i} k \cdot y},
$$

where $(\theta \cdot k)^{\mu}=\theta^{\mu \nu} k_{\nu}$ and $k \cdot y=k_{\mu} y^{\mu}$.

Since divergences come from singularities due to point like interactions, there was hope that field theories on these deformed algebras may be better behaved. This is not the case. Loop integrals in noncommutative field theories differ from their commutative counterpart by phase factors $\mathrm{e}^{ \pm \frac{1}{2} \mathrm{i} \theta^{\mu \nu} p_{\mu} q_{\nu}}$ [15]. In planar diagrams the resulting phases of inner momenta cancel, i.e. the same integral with the same divergences occurs as in undeformed models.

Next question concerns renormalizability: By direct evaluation of Feynman graphs it was shown that Yang-Mills theory is one-loop renormalizable, i.e. the divergences can be absorbed such that Ward-Slavnov identities are fulfilled [16, 17]. For higher loop contributions the new phenomenon of UV/IR-mixing occurs, which was first discovered for scalar fields [18] and shortly later for Yang-Mills theories as well [19]. Although nonplanar one-loop diagrams are convergent for generic external momenta, they diverge for exceptional external momenta, where the regulating phase becomes inefficient. Inserted 
as subgraphs into a bigger graph, the external momenta of the subgraphs are internal momenta for the bigger graph, and the exceptional momenta are realized by the loop integration. The total graph thus becomes divergent. Due to the integrated phase factors involving inner momenta of non-planar graphs, the resulting counterterms are of different structure than the initial action was: the model is not renormalizable.

The problem was analyzed to all loop orders by Chepelev and Roiban [20, 21]. They represented the integrals of noncommutative field theories by ribbon graphs drawn on a genus- $g$ Riemann surface with boundary and proved a power-counting theorem for such a situation. The conclusion is that non-planar graphs are convergent except for two dangerous cases. So called rings stacked on the came cycle lead (in four dimensions) to singularities of arbitrarily high order. There are proposals to reorder the perturbation series in order to sum up these divergences. Commutants occur if exceptional momenta are forced by momentum conservation for subgraphs. They lead to non-local counterterms.

One possible way out concerns additional symmetries. We used a superfield formulation and established a proof that the Wess-Zumino model does not have the above mentioned disease [22]. Since only logarithmically divergent diagrams occur, and we showed earlier that even iterated integrals of this type are integrable, the standard renormalizability proof may be adapted.

\subsection{Seiberg-Witten map}

The phase factors $\mathrm{e}^{ \pm \frac{1}{2} \mathrm{i} \theta^{\mu \nu} p_{\mu} q_{\nu}}$ were kept intact in the previous treatment. There is also the possibility of expanding the phases into a Taylor series in $\theta$. Limiting the expansion to some finite order in $\theta$, we actually get a local (commutative) field theory involving higher-order partial derivatives of the fields. For example, limiting the Taylor expansion of the noncommutative Maxwell action

$$
\Gamma=-\frac{1}{4 g^{2}} \int \hat{F}_{\mu \nu} \star \hat{F}^{\mu \nu}, \quad \hat{F}_{\mu \nu}=\partial_{\mu} \hat{A}_{\nu}-\partial_{\nu} \hat{A}_{\mu}-\mathrm{i} \hat{A}_{\mu} \star \hat{A}_{\nu}+\mathrm{i} \hat{A}_{\nu} \star \hat{A}_{\mu},
$$

to first order in $\theta$, we get

$$
\begin{aligned}
\Gamma & =\int d^{4} x\left(-\frac{1}{4 g^{2}} \tilde{F}_{\mu \nu} \tilde{F}^{\mu \nu}-\frac{1}{2 g^{2}} \theta^{\alpha \beta} \tilde{F}^{\mu \nu} \partial_{\alpha} \hat{A}_{\mu} \partial_{\beta} \hat{A}_{\nu}+\mathcal{O}\left(\theta^{2}\right)\right), \\
\tilde{F}_{\mu \nu} & =\partial_{\mu} \hat{A}_{\nu}-\partial_{\nu} \hat{A}_{\mu} .
\end{aligned}
$$

At higher order in $\theta$ the result of the Taylor expansion becomes extremely complicated.

Guided by an equivalence argument for regularization schemes (point-splitting versus Pauli-Villars), Seiberg and Witten discovered for gauge theories a change of variables so that the $\theta$-expanded action becomes at each order in $\theta$ invariant under commutative gauge transformations [13]. Denote by $\hat{\lambda}, \hat{A}$ and $\hat{F}$ gauge parameter, gauge connection and field strength for the deformed model, and by $\lambda, A$ and $F$ the appropriate quantities for the undeformed case. For the Seiberg-Witten map one requires that

$$
\delta_{\lambda[\hat{\lambda}, A]} \hat{A}[A]=\hat{A}\left[\delta_{\lambda} A\right],
$$

which means that the gauge transformed noncommutative field should be equal to the noncommutative field of the gauge transformed commutative one. The requirement (3.7) 
can be reformulated as a differential equation $\frac{d \hat{A}_{\mu}}{d \theta^{\alpha \beta}}=f_{\mu \alpha \beta}[\hat{A}]$ for some functional $f_{\mu \alpha \beta}$ (see below) and has at first order in $\theta$ the solution

$$
\hat{A}_{\mu}=-\frac{1}{2} \theta^{\alpha \beta} A_{\alpha}\left(\partial_{\beta} A_{\mu}+F_{\beta \mu}\right)+\mathcal{O}(\theta), \quad F_{\mu \nu}=\partial_{\mu} A_{\nu}-\partial_{\nu} A_{\mu} .
$$

Inserting (3.8) into (3.6) one gets

$$
\Gamma=\int d^{4} x\left(-\frac{1}{4 g^{2}} F_{\mu \nu} F^{\mu \nu}-\frac{1}{2 g^{2}} \theta^{\alpha \beta}\left(F_{\alpha \mu} F_{\beta \nu} F^{\mu \nu}-\frac{1}{4} F_{\alpha \beta} F_{\mu \nu} F^{\mu \nu}\right)+\mathcal{O}\left(\theta^{2}\right)\right) .
$$

At higher order in $\theta$ the requirement (3.7) leaves a big amount of freedom open. Various methods are available to get the mapping from $A$ to $\hat{A}$, or better to get the expansion of the latter in terms of $A$. This map is meant as a formal power expansion series in $\theta^{\mu \nu}$. The Munich group $[23,24,25]$ used these ideas and argued that this is the way to obtain a finite number of degrees of freedom in non-Abelian noncommutative Yang-Mills theories.

The Seiberg-Witten map of the noncommutative Yang-Mills action leads to a gauge field theory with an infinite number of vertices and Feynman graphs with unbounded degree of divergences, which seems to rule out a perturbative renormalization. The advantage is, however, that there cannot be any UV/IR-mixing. An explicit quantum field theoretical investigation [26] of the action (3.9) led to the surprising result that the divergent terms of the one-loop photon self-energy are gauge-invariant, independent of the choice of a linear or non-linear gauge and independent of the gauge parameter. These terms could not be absorbed in a standard wave function renormalization. We have thus interpreted our result as the need to extend the classical action by terms of order $\theta^{2}$.

Shortly later we found a possibility to absorb the divergences of the photon self-energy to any loop order and any order in $\theta$ through a more general type of wave function renormalization - a field redefinition [27]. This possibility is related to the freedom in the Seiberg-Witten map. For noncommutative photons $\hat{A}_{\mu}$,

$$
\left(\frac{d \hat{A}_{\mu}}{d \theta^{\rho \sigma}}\right)^{\prime}=\frac{d \hat{A}_{\mu}}{d \theta^{\rho \sigma}}+\Omega_{\rho \sigma \mu}, \quad \Omega_{\rho \sigma \mu} \text { covariant }
$$

is an admissible Seiberg-Witten differential equation if $\frac{d \hat{A}_{\mu}}{d \theta^{\rho \sigma}}$ is. If $\Gamma^{(n)}$ is the order- $n$ term of the $\theta$-expansion of the noncommutative Maxwell action (3.5), and if $\Omega_{\rho \sigma \mu}$ contains explicitly $n-1$ factors of $\theta$, we have

$$
\left(\Gamma^{(n)}\right)^{\prime}-\Gamma^{(n)}=\frac{1}{g^{2}} \int d^{4} x \theta^{\rho \sigma} \Omega_{\rho \sigma \mu}^{(n-1)} \partial_{\nu} F^{\nu \mu} .
$$

It is not difficult to show that any counterterm to the two-point function of $\theta$-expanded Maxwell theory has the form (3.11) so that by a suitable choice of $\Omega$ we can remove all superficial divergences.

This tells us that field redefinitions are capable to absorb a considerable amount of divergences in $\theta$-expanded noncommutative field theories. There was even some hope that $\theta$-expanded field theories may be renormalisable. The crucial question for renormalisability is whether the freedom due to field redefinitions is big enough to absorb 
all divergences. A direct calculation of the 3-point function at lowest order $\theta^{3}$ leads to $\sim 200000$ different contributions which are impossible to analyse. We have therefore tried to find symmetries of the $\theta$-expanded action which limit the structure of possible divergences. The existence of such symmetries is already suggested by (3.9) where the relative coefficient between the two possible gauge invariants of dimension 4 linear in $\theta$, which are $\theta^{\alpha \beta} F_{\alpha \mu} F_{\beta \nu} F^{\mu \nu}$ and $\theta^{\alpha \beta} F_{\alpha \beta} F_{\mu \nu} F^{\mu \nu}$, is $-\frac{1}{4}$. This particular combination seems to be related to the energy-momentum tensor so that one could expect that the $\theta$-expansion of noncommutative Lorentz transformations provides the symmetries looked for.

We have proved in [28] that the $\theta$-expansion reduces the noncommutative Lorentz transformations to a combination of commutative gauge transformations and commutative Lorentz transformations and thus gives no hints at all for new symmetries. As a by-product, however, we have found a new interpretation of the Seiberg-Witten map. We achieved a different kind of $\theta$-expansion which unless Taylor expansion and change of variables which pass through (3.6) allow us to derive (3.9) in a single stroke from (3.5). This derivation is closely related to a deeper discussion of noncommutative Lorentz transformations.

One has to distinguish between two kinds of Lorentz (or more general, conformal) transformations. Lorentz transformations in special relativity relate physical observations made in two inertial reference frames characterised by different velocities and orientations. These transformations can be implemented as coordinate changes, known as $o b$ server Lorentz transformations. Alternatively one considers transformations which relate physical properties of two particles with different helicities or momenta within one specific inertial frame. These are known as particle Lorentz transformations. Usually (without background) these two approaches are equivalent. However, in the presence of a background tensor field this equivalence fails, because the background field will transform as a tensor under observer Lorentz transformation and as a set of scalars under particle Lorentz transformations. We demand

1. that the physical action is invariant under observer Lorentz transformations,

2. that the particle Lorentz transformation (which is not a symmetry) of the action is gauge invariant (thus observable).

From these requirements we derive in [28] that the particle Lorentz transformation of a field is the sum of its naïve observer Lorentz transformation and an additional part given by the Seiberg-Witten differential equation (which is very conveniently derived in this manner).

Since the search for symmetries of the $\theta$-expanded action was not successful, we continued with the direct computation of one-loop divergent graphs. The simplest model to study other Green's functions than the self-energy is $\theta$-deformed QED. For massless electrons there are 9 independent gauge and Lorentz invariant field polynomials of first order in $\theta$. There are 5 independent field redefinitions so that one should expect 4 types of divergences linear in $\theta$ which cannot be removed. The actual one-loop computation of these Green's function in [29] showed that there is only a single independent one-loop divergent Green's functions at first order in $\theta$. In the massive case (where the mass term is inserted directly into the Dirac action) there are 15 invariant terms, 8 field redefinitions and 3 divergences. The computation was very complicated due to the big number of dif- 
ferent graphs involving and the (compared with standard QFT computations) high degree of divergence, which results in contributions from the entire Clifford algebra of $\gamma$ matrices.

Thus, although these computations made clear that $\theta$-expanded field theories can not be expected to be renormalisable, there is evidence now that additional symmetries for the $\theta$-expanded action exist indeed. It is natural to assume that these symmetries are already present in the original noncommutative action before $\theta$-expansion. One possible source is the spectral origin of the action [6] which might admit a bigger symmetry group than just Lorentz and gauge transformations.

The loop calculations of $[26,29]$ were performed for the $\theta$-expanded action after application of the Seiberg-Witten map such as (3.9). As we have shown in [30], very similar computations are possible when starting directly from the Taylor expansion of the action functional such as (3.6). The only difference is that now the gauge symmetry is non-linearly realised so that the whole machinery of external fields and Slavnov-Taylor identities must be used. In other words, one has to extend the gauge-fixed noncommutative QED action by

$$
\Gamma_{\text {ext }}=\int d^{4} x\left(\hat{\bar{\rho}}(\hat{s} \hat{\psi})+(\hat{s} \hat{\bar{\psi}}) \hat{\rho}+\hat{\sigma}^{\mu}\left(\hat{s} \hat{A}_{\mu}\right)+\hat{\kappa}(\hat{s} \hat{c})\right)
$$

where we define the non-commutative BRST-transformations by

$$
\begin{aligned}
\hat{s} \hat{A}_{\mu} & =\hat{D}_{\mu}^{a d j} \hat{c}, & \hat{s} \hat{c} & =\mathrm{i} \hat{c} \star \hat{c}, \\
\hat{s} \hat{\psi} & =\mathrm{i} \hat{c} \star \hat{\psi}, & \hat{\bar{\psi}} & =-\mathrm{i} \hat{\bar{\psi}} \star \hat{c}, \\
\hat{s} \hat{\bar{c}} & =\hat{B}, & \hat{s} \hat{B} & =0 .
\end{aligned}
$$

We denote by $\hat{A}, \hat{\psi}, \hat{c}$ the noncommutative photon, electron and ghost, respectively, and $\hat{\rho}, \hat{\sigma}, \hat{\kappa}$ are the external fields. Loop calculations do not preserve the $\theta$-expansion of (3.13). The Slavnov-Taylor identity

$$
\mathcal{S}(\Gamma)=\int d^{4} x\left(\frac{\delta \Gamma}{\delta \hat{\sigma}^{\mu}} \frac{\delta \Gamma}{\delta \hat{A}_{\mu}}+\frac{\delta \Gamma}{\delta \hat{\kappa}} \frac{\delta \Gamma}{\delta \hat{c}}+\frac{\delta \Gamma}{\delta \hat{\rho}} \frac{\delta \Gamma}{\delta \hat{\bar{\psi}}}+\frac{\delta \Gamma}{\delta \hat{\psi}} \frac{\delta \Gamma}{\delta \hat{\bar{\rho}}}+\hat{B} \frac{\delta \Gamma}{\delta \hat{\bar{c}}}\right)=0
$$

is preserved, though. The identity (3.14) contains several individual identities when differentiating which respect to the fields. To our great surprise we found in [30] that up to first order in $\theta$ and first loop order the Taylor expansion of the noncommutative QED leads up to field redefinition to the same result when using the Seiberg-Witten map or not: There is for massless electrons only a single resulting divergence

$$
\int d^{4} x \mathrm{i} \theta^{\alpha \beta} \hat{\bar{\psi}} \star \gamma^{\mu \nu} \hat{D}_{\beta}^{a d j} \hat{F}_{\mu \nu} \star \hat{\psi}, \quad \hat{D}_{\beta}^{a d j} \hat{F}_{\mu \nu}=\partial_{\beta} \hat{F}_{\mu \nu}-\mathrm{i}\left[\hat{A}_{\beta}, \hat{F}_{\mu \nu}\right]_{\star},
$$

with the numerical coefficient of the divergent term being independent of the application or omission of the Seiberg-Witten map. This seems to indicate that the Seiberg-Witten map is an unphysical change of variables also on quantum level.

This is true to some extent, but there is a subtlety. One can perform the change of variables before or after quantisation. Changing the variables $\Phi^{\prime}=\Phi^{\prime}[\Phi]$ after quantisation, i.e. performing a change of the dummy integration variables in the path integral, one obtains exactly the same Green's functions. The changes in the Feynman rules from $\Phi^{\prime}$ 
to $\Phi$ are compensated by graphs involving the modified source term $\left\langle J, \Phi^{\prime}[\Phi]\right\rangle$ in the path integral. In principle one would also expect contributions from field redefinition ghosts, but here the propagator equals 1 so that there is no contribution at least for certain regularisation schemes. On the other hand, changing the variables in the action functional before inserting it into the path integral, the outcome is expected to be different. However, at first order in $\theta$ only, the difference to the other method is a field redefinition.

Acknowledgement. We would like to thank J. Madore, H. Steinacker, M. Schweda and his group for enjoyable collaborations and the organisers of this school for invitation.

\section{References}

[1] H. Grosse and J. Madore, "A Noncommutative version of the Schwinger model," Phys. Lett. B 283 (1992) 218.

[2] S. Doplicher, K. Fredenhagen and J. E. Roberts, "The Quantum Structure Of Space-Time At The Planck Scale And Quantum Fields," Commun. Math. Phys. 172 (1995) 187 [arXiv:hepth/0303037].

[3] A. Connes, "Noncommutative Geometry," Academic Press, San Diego 1994.

[4] J. Madore, "An introduction to noncommutative differential geometry and its physical applications," Cambridge University Press, 1999.

[5] J. M. Gracia-Bondía, J. C. Várilly and H. Figueroa, "Elements of noncommutative geometry," Birkhäuser, Boston 2000.

[6] A. H. Chamseddine and A. Connes, "The spectral action principle," Commun. Math. Phys. 186 (1997) 731 [arXiv:hep-th/9606001].

[7] H. Grosse, C. Klimčik, and P. Presnajder, "Towards finite quantum field theory in noncommutative geometry," Int. J. Theor. Phys. 35 (1996) 231 [arXiv:hep-th/9505175].

[8] H. Grosse, C. Klimčik, and P. Presnajder, "Topologically nontrivial field configurations in noncommutative geometry," Commun. Math. Phys. 178 (1996) 507 [arXiv:hep-th/9510083].

[9] H. Grosse, C. Klimčik, and P. Presnajder, "Field theory on a supersymmetric lattice," Commun. Math. Phys. 185 (1997) 155 [arXiv:hep-th/9507074].

[10] H. Grosse, J. Madore and H. Steinacker, "Field theory on the $q$-deformed fuzzy sphere. I," J. Geom. Phys. 38 (2001) 308 [arXiv:hep-th/0005273].

[11] H. Grosse, J. Madore and H. Steinacker, "Field theory on the $q$-deformed fuzzy sphere. II: Quantization," J. Geom. Phys. 43 (2002) 205 [arXiv:hep-th/0103164].

[12] A. Y. Alekseev, A. Recknagel and V. Schomerus, "Brane dynamics in background fluxes and non-commutative geometry," JHEP 0005 (2000) 010 [arXiv:hep-th/0003187].

[13] N. Seiberg and E. Witten, "String theory and noncommutative geometry," JHEP 9909 (1999) 032 [arXiv:hep-th/9908142].

[14] A. Connes, M. R. Douglas and A. Schwarz, "Noncommutative geometry and matrix theory: Compactification on tori," JHEP 9802 (1998) 003 [arXiv:hep-th/9711162].

[15] T. Filk, "Divergencies in a field theory on quantum space," Phys. Lett. B 376 (1996) 53. 
[16] C. P. Martín and D. Sánchez-Ruiz, "The one-loop UV divergent structure of U(1) Yang-Mills theory on noncommutative $\mathbb{R}^{4}$," Phys. Rev. Lett. 83 (1999) 476 [arXiv:hep-th/9903077].

[17] T. Krajewski and R. Wulkenhaar, "Perturbative quantum gauge fields on the noncommutative torus," Int. J. Mod. Phys. A 15 (2000) 1011 [arXiv:hep-th/9903187].

[18] S. Minwalla, M. Van Raamsdonk and N. Seiberg, "Noncommutative perturbative dynamics," JHEP 0002 (2000) 020 [arXiv:hep-th/9912072].

[19] A. Matusis, L. Susskind and N. Toumbas, "The IR/UV connection in the non-commutative gauge theories," JHEP 0012 (2000) 002 [arXiv:hep-th/0002075].

[20] I. Chepelev and R. Roiban, "Renormalization of quantum field theories on noncommutative $\mathbb{R}^{d}$. I: Scalars," JHEP 0005 (2000) 037 [arXiv:hep-th/9911098].

[21] I. Chepelev and R. Roiban, "Convergence theorem for non-commutative Feynman graphs and renormalization," JHEP 0103 (2001) 001 [arXiv:hep-th/0008090].

[22] A. A. Bichl, J. M. Grimstrup, H. Grosse, L. Popp, M. Schweda and R. Wulkenhaar, "The superfield formalism applied to the noncommutative Wess-Zumino model," JHEP 0010 (2000) 046 [hep-th/0007050].

[23] B. Jurčo, S. Schraml, P. Schupp and J. Wess, "Enveloping algebra valued gauge transformations for non-Abelian gauge groups on non-commutative spaces," Eur. Phys. J. C 17 (2000) 521 [hep-th/0006246].

[24] J. Madore, S. Schraml, P. Schupp and J. Wess, "Gauge theory on noncommutative spaces," Eur. Phys. J. C 16 (2000) 161 [hep-th/0001203].

[25] B. Jurčo, P. Schupp and J. Wess, "Nonabelian noncommutative gauge theory via noncommutative extra dimensions," Nucl. Phys. B 604 (2001) 148 [hep-th/0102129].

[26] A. A. Bichl, J. M. Grimstrup, L. Popp, M. Schweda and R. Wulkenhaar, "Perturbative analysis of the Seiberg-Witten map," Int. J. Mod. Phys. A 17 (2002) 2219 [hep-th/0102044].

[27] A. Bichl, J. Grimstrup, H. Grosse, L. Popp, M. Schweda and R. Wulkenhaar, "Renormalization of the noncommutative photon selfenergy to all orders via Seiberg-Witten map," JHEP 0106 (2001) 013 [arXiv:hep-th/0104097].

[28] A. A. Bichl, J. M. Grimstrup, H. Grosse, E. Kraus, L. Popp, M. Schweda and R. Wulkenhaar, "Noncommutative Lorentz symmetry and the origin of the Seiberg-Witten map," Eur. Phys. J. C 24 (2002) 165 [arXiv:hep-th/0108045].

[29] R. Wulkenhaar, "Non-renormalizability of $\theta$-expanded noncommutative QED," JHEP 0203 (2002) 024 [arXiv:hep-th/0112248].

[30] J. M. Grimstrup and R. Wulkenhaar, "Quantisation of theta-expanded non-commutative QED," Eur. Phys. J. C 26 (2002) 139 [arXiv:hep-th/0205153]. 